\title{
AN ANALYSIS OF SECURITY CHALLENGES IN MOBILE AD HOC NETWORKS
}

\author{
Ali Dorri and Seyed Reza Kamel and Esmail kheyrkhah \\ Department of Computer Engineering, Mashhad branch, \\ Islamic Azad University, Mashhad, Iran. \\ Alidorri@mshdiau.ac.ir \\ rezakamelecomputer.org \\ e.kheirkhah@mshdiau.ac.ir
}

\begin{abstract}
Mobile Ad Hoc Network (MANET) is a collection of wireless mobile nodes with restricted transmission range and resources, no fixed infrastructure and quick and easy setup. Because of special characteristics, wide-spread deployment of MANET faced lots of challenges like security, routing and clustering. The security challenges arise due to MANETs selfconfiguration and self-maintenance capabilities. In this paper, we present an elaborate view of issues in MANET security. We discussed both security services and attacks in detail. Three important parameters in MANET security are defined. Each attack has been analyses briefly based on its own characteristics and behaviour. In addition, defeating approaches against attacks have been evaluated in some important metrics. After analyses and evaluations, future scopes of work have been presented.
\end{abstract}

\section{KEYWORDS}

Mobile Ad Hoc Network (MANET), Security, Attacks on MANET, Security services, Survey.

\section{INTRODUCTION}

In these years, progresses of wireless technology and increasing popularity of wireless devices, made wireless networks so popular. Mobile Ad Hoc Network (MANET) is an infrastructureindependent network with wireless mobile nodes. MANET is a kind of Ad Hoc networks with special characteristics like open network boundary, dynamic topology, distributed network, fast and quick implementation and hop by hop communications. These characteristics of MANET made it popular, especially in military and disaster management applications. Besides providing benefits, MANET features made it challengeable. Peer to peer applications [1], integration with internet [2], security [3], maintaining network topology [4] and energy [5, 6] are some of the most important challenges in MANET. We briefly discussed MANET challenges in our previous work [7].

In MANET all nodes are free to join and leave the network, also called open network boundary. All intermediate nodes between a source and destination take part in routing, also called hop by hop communications. As communication media is wireless, each node will receive packets in its wireless range, either it has been packets destination or not. These characteristics of MANET increase its vulnerability against malicious behaviours. Therefore, security became the most important challenge in MANET [8].

Natarajan Meghanathan et al. (Eds) : WiMONe, NCS, SPM, CSEIT - 2014

pp. 13-25, 2014. (C) CS \& IT-CSCP 2014

DOI : $10.5121 /$ csit.2014.41202 
In this paper our goal is to provide a comprehensive review on MANET security. Important parameters in security are introduced. Security is divided in two aspects: security services and attacks. A comprehensive review in both security aspects is presented. In addition, we analyses each attack's behaviour in different parameters. Defeating approaches are evaluated in important metrics. Finally, future scopes of work are presented. Rest of this paper is organized as follow: in section 2, three important security parameters in MANET are presented. Section 3 presents two important aspects of security with a discussion on their strategies. Three combinational challenges with security are presented in section 4 . Section 5 presents our analyses and classifications on security of MANET and presents some research interest in security. Section 6 introduces open research issues and directions of researches in MANET security. Finally section 7 concludes the paper and introduces best ways to secure MANET and presents some future works.

\section{IMPORTANT PARAMETERS IN MANET SECURITY}

Because of MANET's special characteristics, there are some important metrics in MANET security that are important in all security approaches; we call them "security parameters". These parameters defined due to MANET special characteristics. Being unaware of these parameters may cause a security approach useless in MANET. Figure 1 shows the relation between security parameters and security challenges. Each security approach must be aware of security parameters as shown in Figure 1. All mechanisms proposed for security aspects, must be aware of these parameters and don't disregard them, otherwise they may be useless in MANET. Security parameters in MANET are as follows:

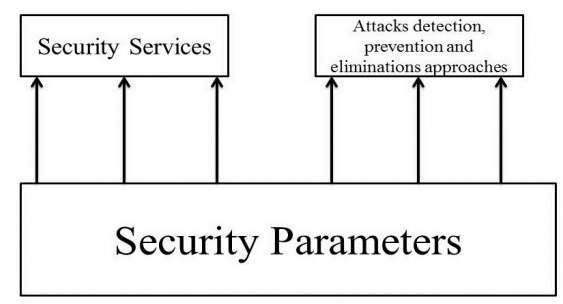

Figure 1.Relation between Security Parameters and Security aspects

Network overhead: Each security mechanism uses a number of control packets. This parameter refers to number of control packets generated by security mechanisms. As MANET uses wireless communications, increasing network overhead may increase collision, congestion and packet loss that may increase packet retransmission.

Processing time: This parameter refers to delay caused by security approach. Because of MANET dynamic topology, neighbours of each node may change in a period of time. As a result, previous information will not be efficient any more.

Energy consumption: Nodes have limited energy resources. One of the major issues is the limited energy, which is usually supplied by node batteries that network nodes possess. Therefore security approach must have low energy consumption.

Each security protocol must be aware of these three important parameters. In some situations a trade-off between these parameters is provided in order to perform a satisfaction level in all of them. Security protocols that disregard these parameters aren't efficient as they waste network resources. 


\section{MANET SECURITY CHALLENGES}

One of the earliest researches in security in MANET was presented in 2002 [9]. Some security challenges in MANET were inherited from ad hoc networks that were research interests since 1999 [10, 11]. Generally there are two important aspects in security: Security services and Attacks. Services refer to some protecting policies in order to make a secure network, while attacks use network vulnerabilities to defeat a security service. In the next two parts, we will discuss these two important aspects of MANET security.

\subsection{Security Services}

Security services are used to secure a network. Because of special features of MANET, providing these services is a great challenge. For securing MANET a trade-off between these services must be provided, which means if one service guarantees without noticing other services, security system will fail. Providing a trade-off between these security services is depended on network application, but the problem is to provide services one by one in MANET and presenting a way to guarantee each service. We discuss five important security services and their challenges as follows:

Availability: According to this service, each authorized node must have access to all data and services in the network. Availability challenges arise due to MANETs dynamic topology and open boundary. To provide this service, each authorized node must have access to data and services when it needs them. Time spent for accessing is important as time is one of security parameters. By using lots of security and authentication levels, this service is disregarded as passing security levels needs time. Existing approaches for providing this service aren't efficient in MANET and by using them, high degree of distribution, autonomy and dynamicity of MANET will be lost [12]. Authors in [12] provided a new way to solve this problem by using a new trust based clustering approach. In the proposed approach which is called ABTMC (Availability Based Trust Model of Clusters), by using availability based trust model, hostile nodes are identified quickly and should be isolated from the network in a period of time, therefore availability of MANET will be guaranteed.

Authentication: The goal of this service is to provide trustable communications between two different nodes. When a node receives packets from a source, it must be sure about identity of the source node. One way to provide this service is using certifications, whoever in absence of central control unit, key distribution and key management are challengeable. In [13] the authors presented a new way based on trust model and clustering to public the certificate keys. In this case, the network is divided into some clusters and in this clusters public key distribution will be safe by mechanisms provided in the paper. Their simulation results show that, the presented approach is better than PGP. But it has some limitations like clustering. MANET dynamic topology and unpredictable nodes position, made clustering challengeable.

Data confidentially: According to this service, each node or application must have access to specified services that it has the permission to access. Most of services that are provided by data confidentially use encryption methods but in MANET as there is no central management, key distribution faced lots of challenges and in some cases impossible. Authors in [14] proposed a new scheme for reliable data delivery to enhance the data confidentially. The basic idea is to transform a secret message into multiple shares by secret sharing schemes and then deliver the shares via multiple independent paths to the destination. Therefore, even if a small number of nodes that are used to relay the message shares, been compromised, the secret message as a whole is not compromised. Also this way protects network, but packet delivery delay will be increased. 
The reason is that, data is sent in multipath. Using multipath delivering causes the variation of delay in packet delivery for different packets. It also leads to out-of-order packet delivery.

Integrity: According to integrity, just authorized nodes can create, edit or delete packets. As an example, Man-In-The-Middle attack is against this service. In this attack, the attacker captures all packets and then removes or modifies them. Authors in [15] presented a mechanism to modify the DSR routing protocol and gain to data integrity by securing the discovering phase of routing protocol.

Non-Repudiation: By using this service, neither source nor destination can repudiate thier behaviour or data. In other words, if a node receives a packet from node 2, and sends a reply, node 2 cannot repudiate the packet that it has been sent. Authors in [16] presented a new approach that is based on grouping and limiting hops in broadcast packets. All group members have a private key to ensure that another node couldn't create packets with its properties. But creating groups in MANET is challengeable.

Till now we discussed security services challenges in MANET. Detecting and eliminating malicious nodes, is another aspect of the MANET security. In the next section, we will discuss some important attacks in MANET and ways to detect and eliminate them.

\subsection{Attacks}

As described before, MANET has lots of characteristics like hop by hop communications, wireless media, open border and easy to setup, that made it popular. whoever these characteristics made it popular for malicious nodes. Here are some important attacks in MANET:

Black Hole Attack: In this attack, malicious node injects fault routing information to the network and leads packets toward itself, then discards all of them [17-19]. We presented a comprehensive review in this attack in our previous work [20]. We present some ways to detect and eliminate black hole nodes, also we present some classifications in black hole. In [21] authors presented a new approach to detect black hole by sending packets in shared paths and also fetching sequence number of packets. This attack is against routing and is depended on routing protocol.

Worm Hole Attack: In worm hole attack, an attacker records packets at one location of network and tunnels them to another location [22]. Fault routing information could disrupt routes in network [23]. Authors in [24] presented a way to secure MANET against this attack by using encryption and node location information. But as mentioned before, key distribution is a challenge in MANET.

Byzantine attack: In this attack, malicious node injects fault routing information to network, in order to locate packets into a loop [25, 26]. One way to protect network against this attack is using authentication. Authors in [27] presented a mechanism to defeat against this attack using RSA authentication.

Snooping attack: The goal of this attack is accessing to other nodes packets without permission [28]. As in MANET packets transmitted hop by hop, any malicious node can capture others packets.

Routing attack: In this attack, malicious nodes try to modify or delete node's routing tables [17, $18,29]$. Using this attack, malicious nodes destroy routing information. Therefore, packet overhead and processing time will increase. 
Resource consumption attack: The goal of this attack is wasting network or node's resources $[30,31]$. As a way to do this, malicious nodes lead packets to a loop. Therefore, nodes resources consume so fast.

Session hijacking: Session hijacking is a critical error and gives an opportunity to the malicious node to behave as a legitimate system [32,33]. Using this attack, malicious node reacts instead of true node in communications. Cryptography is one of the most efficient ways to defeat this attack.

Denial of service: In this attack, malicious node prevents other authorized nodes to access network data or services [34-38]. Using this attack, a specific node or service will be inaccessible and network resources like bandwidth will be wasted. In addition, packet delay and congestion increases.

Jamming attack: Jamming attack is a kind of DOS attack [39]. The objective of a jammer is to interfere with legitimate wireless communications. A jammer can achieve this goal by either preventing a real traffic source from sending out a packet, or by preventing the reception of legitimate packets [40].

Impersonation Attack: Using this attack, attacker can pretend itself as another node and injects fault information to the network [41-43]. As MANET has open border and hop-by-hop communications, it's hardly vulnerable against this attack. In some cases even using authentication is useless.

Modification Attack: In this attack, malicious nodes sniff the network for a period of time. Then, explore wireless frequency and use it to modify packets [44, 45]. Man-in-the-middle is a kind of Modification attack.

Fabrication Attack: In fabrication attack, malicious node destroys routing table of nodes by injecting fault information [46-48]. Malicious node creates fault routing paths. As a result, nodes send their packets in fault routes. Therefore, network resources wasted, packet delivery rate decreased and packet lost will growth.

Man-in-the-middle attack: In this attack, malicious node puts itself between source and destination. Then, captures all packets and drops or modifies them [49-51]. Hop by hop communications are made MANET vulnerable against this attack. Authentication and cryptography are the most effective ways to defeat this attack.

Gray Hole Attack: This attack is similar to black hole. In black hole, malicious node drops all packets, while in this attack, malicious node drops packets with different probabilities [52-55]. As it relays some packets, detecting this attack is more complicated than black hole and some detection approaches like sniffing or watchdog will be useless in it.

Traffic Analyse Attack: The goal of this attack is sniffing network traffic to use them in another attack or in a specific time [44, 56]. Malicious node captures all packets to use them later. 


\section{INCORPORATING SECURITY AND OTHER CHALLENGES}

One way to provide security in MANET, besides decreasing network overhead, is to incorporate security approaches with other challenges. In this way, both challenges are solved by improving security parameters in total. We discuss these combinational approaches as follows:

Secure routing protocols: Using this method, security will be guaranteed in routing phase. When a node wants to create a path to a destination, it uses some mechanisms to select a secure path. Then malicious nodes will be detected and eliminated. Authors in [57] presented a secure routing protocol based on using IPSEC in MANET routing protocols. Authors in [58] presented a trust based security routing protocol to create secure path. In MANET, there is more than one path between two different nodes. Selecting best path based on both routing and security, will improve security parameters.

Security in QOS: Providing security has negative impact on QOS. Therefore, providing QOS beside security is important. Authors in [59] presented a game theory to make a trade-off between security and QOS. Authors in [60] provided an approach that creates QOS aware multipath between source and destination with link information. By providing security in QOS, a level of security and QOS will be guaranteed with low time or network overhead.

Cluster-based Security: These mechanisms use clustering in network to provide more efficient situations for security protocols. Generally in these methods, clusters are used for key distribution or as central management. Using clustering for security goals is important as it could solve problems in key distribution or key management. Authors in [61, 62] provides a key distribution mechanism by using clusters. Using clusters make it easy for security mechanisms, but grouping nodes in clusters and maintaining clusters, are challengeable.

As another benefit of these combinational approaches, there should be a trade-off between challenges based on each one's importance. Based on application, one challenge may be more important than the other one. Then, an algorithm may be proposed with better security parameters. For example, when selecting best path in routing is not as important as security, an approach can choose more secure paths without emphasizing on best routing path. As an example, in AODV routing protocol, the path with low sequence number is chosen as the best path. The reason is that, if there be a malicious node, it will send high sequence number.

\section{ANALYSES AND DISCUSSION}

Previous sections discussed attacks and security services in MANET. This section presents an analytics and classification on previous issues. In order to analyses attacks and their behaviour, we presented analyses in each attack in Table 1. For each attack five important parameters has been discussed. These parameters are as follows:

- Violated Service: Each attack breaks a security service. We presented the most important defeated service in this column.

- The Proposed Solutions: Some of the most effective approaches to detect and eliminate malicious nodes. 
- MANET features which lead to this attack: Each malicious node uses a feature or features of MANET to break the security.

- Attack Type: Lots of researches classified attacks in two mainly class that are as follows: Active attack, Passive attack. In passive attacks, malicious node listens to transmissions without any active injection or effect on network[63]. While, in active attacks malicious node inject information.

- Attack Goal: The most important goal of each attack.

From Table 1it's obvious that lots of attacks are against availability. In availability aimed attacks, malicious nodes inject fault routing information or destroy nodes routing tables in order to defeat availability. As another point in Table 1, sniffing is one of the widely used mechanisms to defeat against attacks. Generally in sniffing mechanisms, sniffer put it-self in promiscuous mode and listen to the network traffic. In this way, it can detect misbehaviour of malicious nodes. As another effective defeating approach, we can name encryption and route information. Encryption mechanism defeat packets against accessing or modification. Malicious nodes can't modify encrypted packets. Whoever, they can drop packets and break availability. Routing information in MANET is nightly variable. Routing information approach, secure MANET using additional controller packets. There are six different defeating mechanisms as presented in Table 1. Defeating approaches are important in MANETs security. Therefore, an analytics on these approaches is presented in Table 2 .

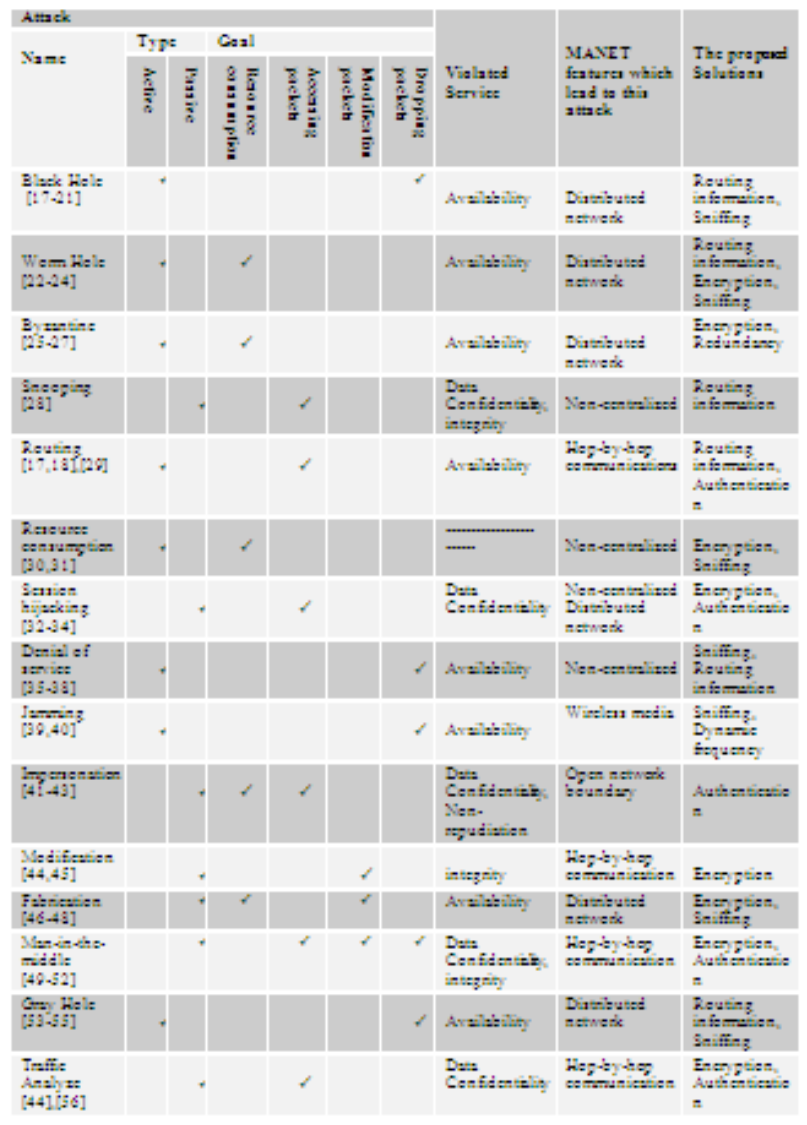

Table 1: Analytics on MANET Attacks. 
Each proposed solution uses some of network resources in order to detect an attack. Table 2 presents five important parameters for security mechanisms and discussed the effect of each proposed solution in parameters. In order to analyses the energy consumption of approaches; we compare energy consumption in each approach toward others. The word 'low' means it consumes lower energy than other approaches. Also, accuracy refers to ability in defeating single or cooperative malicious nodes. In the case of cooperative attacks, malicious nodes work with each other in order to cover their tracks.

Routing information approach generates controller packets and uses them in order to detect malicious nodes. In addition, in some cases nodes must keep additional routing table like DRI [19]. As mentioned, in sniffing each node must put it-self in promiscuous mode and capture all packets transmission in its range. This feature of sniffing wastes nodes energy. Also it increases process and memory overhead. In the case of cooperative malicious nodes, sniffing is useless as malicious nodes may work with each other to proof them-selves as trustable nodes.

Redundancy and dynamic frequency approaches can't detect the malicious nodes. These approaches can only avoid network from an attack. In the case of misbehaviour, these approaches can detect attack, while they are unable to detect the malicious nodes or eliminate them from whole network. In other work, these approaches can just find the path with malicious nodes.

In routing information, control packets transmission increase processing time. In encryption and authentication, key distribution is an important challenge, because of lack of central infrastructure or key distribution center. Therefore, each malicious node can pretend it-self as a trustable node and take part in key distribution. In redundancy, destination must buffer packets in order to get packets in sequence or to compare them with each other. In addition, it increases traffic overhead by sending duplicated packets. That cause increasing in congestion, packet lost and energy consumption.

In addition of five important parameters, Table 2 presents some limitations on each defeating approaches. These limitations come from each approaches characteristics. These limitations decrease performance or efficiency of each approach.

\section{FUTURE DIRECTIONS OF RESEARCHES}

Until now we briefly discussed the security challenges in MANET and present some analytics in them. In this section we present open research issues.

Routing information approaches are suitable in all types of MANET. In this approach, reducing packet overhead and processing time, beside increasing accuracy is an important challenge. By increasing accuracy, it can detect cooperative malicious nodes. With decreasing processing time of this approach MANETs flexibility will increase.

Sniffing approach is useful in the case of single attacks, as it is unable to detect cooperative nodes. Whoever, it waste nodes energy and it is not suitable in MANET with high speed nodes. Finding a more effective way to calculate the threshold and present effective detection mechanism forasmuch as decreasing time and packet overhead is the open border of research in sniffing approaches. Beside it, detecting cooperative malicious nodes is challengeable. In order to solve this challenge comparing sniffing with other defeating approaches is recommended. 
Table 2: Analysis at the proposed solutions

\begin{tabular}{|c|c|c|c|c|c|c|}
\hline $\begin{array}{l}\text { The proposed } \\
\text { Solutious }\end{array}$ & \begin{tabular}{|l|} 
Energy \\
Cousumption \\
\end{tabular} & $\begin{array}{l}\text { Process } \\
\text { Overhead }\end{array}$ & $\begin{array}{l}\text { Memory } \\
\text { overhead }\end{array}$ & $\begin{array}{l}\text { Packet } \\
\text { Overhead }\end{array}$ & Accuracy & Limitatious \\
\hline $\begin{array}{l}\text { Routing } \\
\text { Information } \\
\text { [191.[25-271.[33] }\end{array}$ & Low & & $\checkmark$ & $\checkmark$ & S,C & $\begin{array}{l}\text { Processing } \\
\text { Time }\end{array}$ \\
\hline Sniffing & High & $\checkmark$ & $\checkmark$ & & S & $\begin{array}{l}\text { Cooperative } \\
\text { nodes }\end{array}$ \\
\hline $\begin{array}{l}\text { Encryption } \\
{[49][51],[61,62]}\end{array}$ & Normal & $\checkmark$ & $\checkmark$ & $\checkmark$ & S,C & $\begin{array}{l}\text { Absence of } \\
\text { Centralized } \\
\text { Control. } \\
\text { Key } \\
\text { distribution }\end{array}$ \\
\hline $\begin{array}{l}\text { Redundancy } \\
\text { [391.[60] }\end{array}$ & High & & & $\checkmark$ & None & $\begin{array}{l}\text { Packet } \\
\text { overhead }\end{array}$ \\
\hline $\begin{array}{l}\text { Authentication } \\
{[41,42]}\end{array}$ & Low & $\checkmark$ & & $\checkmark$ & S & $\begin{array}{l}\text { Absence of } \\
\text { Centralized } \\
\text { Control. } \\
\text { Key } \\
\text { distribution }\end{array}$ \\
\hline $\begin{array}{l}\text { Dynanic } \\
\text { Frequency } \\
{[39,40]}\end{array}$ & High & & & $\checkmark$ & None & $\begin{array}{l}\text { Frequency } \\
\text { knowledge }\end{array}$ \\
\hline $\begin{array}{l}\text { The proposed } \\
\text { Solutious }\end{array}$ & $\begin{array}{l}\text { Euergy } \\
\text { Cousumption }\end{array}$ & $\begin{array}{l}\text { Process } \\
\text { Overhead }\end{array}$ & $\begin{array}{l}\text { Memory } \\
\text { overhesd }\end{array}$ & $\begin{array}{l}\text { Packet } \\
\text { Overhead }\end{array}$ & Accuracy & Limitatious \\
\hline $\begin{array}{l}\text { Routing } \\
\text { Information } \\
\text { [19].[25-27].[33] }\end{array}$ & Low & & $\checkmark$ & $\checkmark$ & $S, C$ & $\begin{array}{l}\text { Processing } \\
\text { Time }\end{array}$ \\
\hline $\begin{array}{l}\text { Sniffing } \\
{[22],[38],[56]}\end{array}$ & High & $\checkmark$ & $\checkmark$ & & S & $\begin{array}{l}\text { Cooperative } \\
\text { nodes }\end{array}$ \\
\hline $\begin{array}{l}\text { Eneryption } \\
{[49][51],[61,62]}\end{array}$ & Normal & $\checkmark$ & $\checkmark$ & $\checkmark$ & S,C & $\begin{array}{l}\text { Absence of } \\
\text { Centralizad } \\
\text { Control. } \\
\text { Key } \\
\text { distribution }\end{array}$ \\
\hline $\begin{array}{l}\text { Redundancy } \\
{[391 .[60]}\end{array}$ & High & & & $\checkmark$ & None & $\begin{array}{l}\text { Packet } \\
\text { overhead }\end{array}$ \\
\hline $\begin{array}{l}\text { Authentication } \\
{[41,42]}\end{array}$ & Low & $\checkmark$ & & $\checkmark$ & S & $\begin{array}{l}\text { Absence of } \\
\text { Centralizad } \\
\text { Control, } \\
\text { Key } \\
\text { distribution }\end{array}$ \\
\hline $\begin{array}{l}\text { Dynanic } \\
\text { Frequency } \\
{[39,40]}\end{array}$ & High & & & $\checkmark$ & None & $\begin{array}{l}\text { Frequency } \\
\text { knowledge }\end{array}$ \\
\hline
\end{tabular}

MANET is self-organized, self-configurable network without any centralized control. Therefore, encryption and authentication are challengeable. Key distribution and control unit are the most important challenges. One way to over through these challenges is using clustering; therefore, the Cluster Head can act as the key distributer. Because of MANETs dynamic topology, creating and maintain clusters is highly challengeable. Using fuzzy logic [64] or swarm based [65] is highly recommended for this challenge. As another research interest, decreasing processing time and processing overhead of encryption approach can be mentioned.

Redundancy approaches, generate lots of duplicated packets and waste nodes resources. Also it increases congestion and packet lost. Effectively choosing number of duplicated paths, based on risk level, is highly challengeable. Also combining this approach with some other approaches in order to detect malicious nodes is another challengeable issue.

Dynamic frequency is effective in multi-type MANETs. By using this approach in multi-type MANET, each node secures its packets by sending in different frequencies. In addition, breaking one frequency has no effect on others. This is a challenge in this approach. 


\section{CONCLUSION}

Mobile Ad Hoc Network (MANET) is a kind of Ad hoc network with mobile, wireless nodes. In MANET, all nodes are free to join and leave the network. Features of MANET like dynamic topology and distributed network, made it popular and also challengeable. Open network boundary, dynamic topology and hop by hop communications made security the most important challenge in MANET.

In this paper, we presented a comprehensive review on security challenges in MANET. We divided security in two aspects: security services and attacks. We discussed each one in detail; also we introduced security parameters that are important in MANET security. Finally some analyses and classifications in security approaches were presented. As a result of our analyses, securing network in routing path using route table information, and encryption, are two efficient ways to secure MANET. Each of these approaches have some benefits and limitations. As military applications are one of MANET's applications, encryption is important to avoid malicious nodes to access data packets.

\section{REFERENCES}

[1] A. Gantes and j. stucky, "A platform on a Mobile Ad hoc Network challenging collaborative gaming," international symposium on collaborative technologies and systems, 2008.

[2] K. U. R. Khan, R. U. Zaman, and A. V. G. Reddy, "Integrating Mobile Ad Hoc Networks and the Internet challenges and a review of strategies," presented at the 3rd International Conference on Communication Systems Software and Middleware and Workshops, COMSWARE, 2008.

[3] M. Suguna and P. Subathra, " Establishment of stable certificate chains for authentication in mobile ad hoc networks," presented at the International Conference on Recent Trends in Information Technology (ICRTIT), 2011.

[4] H. Nishiyama, T. Ngo, N. Ansari, and N. Kato, "On Minimizing the Impact of Mobility on Topology Control in Mobile Ad Hoc Networks," Wireless Communications, IEEE Transactions, 2012.

[5] F. D. Rango, M. Fotino, and S. Marano, "EE-OLSR: Energy Efficient OLSR routing protocol for Mobile ad-hoc Networks," presented at the Military Communications Conference, MILCOM, 2008.

[6] K. Du and Y. Yang, "Policy-Based Time Slot Assignment algorithm in a MANET(PBTSA)," presented at the 3rd International Conference on Anti-counterfeiting, Security, and Identification in Communication, ASID, 2009.

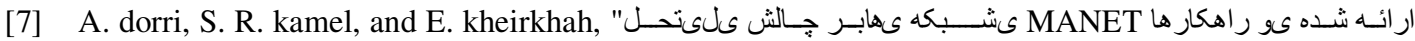
آن presented at the first national conference on electronical and computer north Iran (Bandar Anzali), 2014.

[8] R. Sheikh, M. S. Chande, and D. K. Mishra, "Security issues in MANET:A review," presented at the Seventh International Conference On Wireless And Optical Communications Networks (WOCN), 2010.

[9] H. Deng, W. Li, and D. P. Agrawal, "Routing security in wireless ad hoc networks,," Communications Magazine,IEEE, 2002.

[10] Y.Z.a and W. Lee, "Intrusion Detection in Wireless Ad-Hoc networks," presented at the 6th Int'l. Conf. Mobile Comp. Net., MobiCom, 2000.

[11] F.S.a and R. Anderson, "The Resurrecting Ducking: Security Issues for Ad-Hoc Wireless Networks," 7th Int'l. Wksp on Security Protocols. Proc., LNC, 1999.

[12] X. Zhao, Z. You, Z. Zhao, D. Chen, and F. Peng, "Availability Based Trust Model of Clusters for MANET," presented at the 7th International Conference on Service Systems and Service Management (ICSSSM), 2011.

[13] E. C.H.Ngai and L. M. R, "Trust and clustering-based Authentication Services in Mobile ad hoc networks," presented at the proceeding of the 24th international conference on Distributed Computing systems Workshops 2004.

[14] W. Lou, W. Liu, and Y. Fang, "SPREAD: enhancing data confidentiality in mobile ad hoc networks," presented at the Twenty-third AnnualJoint Conference of the IEEE Computer and Communications Societies, 2004. 
[15] S. Rana and A. Kapil, "Security-Aware Efficient Route Discovery for DSR in MANET," Information and Communication Technologies, Communications in Computer and Information Science, vol. 101, pp. 186-194, 2010.

[16] X. Lv and H. Li, "Secure group communication with both confidentiality and non-repudiation for mobile ad-hoc networks," Information Security, IET, vol. 7, 2013.

[17] S.a.A.k.G, H.o.d.R.m, and S. sharma, "A Comprehensive Review of Security Issues in Manets," International Journal of Computer Applications vol. 692013.

[18] V.P. and R. P. Goyal, "MANET: Vulnerabilities, Challenges, Attacks, Application," IJCEM International journal of Computational Engineering \& management, vol. 11, 2011.

[19] A. MISHRA, R. Jaiswal, and S. Sharma, " A novel approach for detecting and eliminating cooperative black hole attack using advanced DRI table in Ad hoc Network," presented at the 3rd International Conference on Advance Computing Conference (IACC), 2013

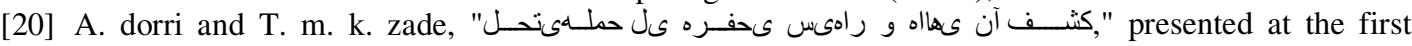
regional conference on optimizing and soft computing in electronic and computer engineering, 2014.

[21] N. S. A. Sharma, "The Black-Hole Node Attack in MANET," presented at the Second International Conference on Advanced Computing \& Communication Technologies, ACCT '12 Proceedings of the, 2012.

[22] M. A. Gorlatova, P. C. Mason, M. Wang, and L. Lamont, " Detecting Wormhole Attacks in Mobile Ad Hoc Networks through Protocol Breaking and Packet Timing Analysis," Military Communications Conference, IEEE, MILCOM, 2006.

[23] S. Keer and A. Suryavanshi, "To prevent wormhole attacks using wireless protocol in MANET," presented at the nternational Conference on Computer and Communication Technology (ICCCT), 2010.

[24] Z. A. Khan and M. H. Islam, "Wormhole attack: A new detection technique," presented at the international conference on Emerging Technologies (ICET), 2012.

[25] M. Yu, M. C. Zhou, and W. Su, "A Secure Routing Protocol Against Byzantine Attacks for MANETs in Adversarial Environments," IEEE Transactions on Vehicular Technology, vol. 58

[26] G. Singla, M. S. Sathisha, A. Ranjan, S. D., and P. Kumara, "Implementation of protected routing to defend byzantine attacks for MANET's," International Journal of Advanced Research in Computer Science, vol. 3, p. 109, 2012.

[27] G. Singla and P. Kaliyar, "A Secure Routing Protocol for MANETs Against Byzantine Attacks," Computer Networks \& Communications (NetCom), Lecture Notes in Electrical Engineering, vol. 131, pp. 571-578, 2013.

[28] S. Shaw, K. Orea, P. Venkateswaran, and R. Nandi, " Simulation and Performance Analysis of OLSR under Identity Spoofing Attack for Mobile Ad-Hoc Networks," Computer Networks and Information Technologies Communications in Computer and Information Science, vol. 142, pp. 308-310, 2011.

[29] B. Kannhavong, H. Nakayama, Y. Nemoto, and N. Kato, "A survey of routing attacks in mobile ad hoc networks," Wireless Communications, IEEE Transactions, vol. 14

[30] M. Abdelhaq, R. Hassan, and R. Alsaqour, "Using Dendritic Cell Algorithm to Detect the Resource Consumption Attack over MANET," Software Engineering and Computer Systems Communications in Computer and Information Science vol. 181, pp. 429-442, 2011.

[31] L. Rajeswari, A. Prema, R. A. Xavier, and A. Kannan, "Enhanced intrusion detection techniques for mobile ad hoc networks," presented at the International Conference on Information and Communication Technology in Electrical Sciences (ICTES), 2007.

[32] A. K. Rai, R. R. Tewari, and S. K. Upadhyay, "different type of attacks on integrated MANETinternet comunication," international jornal of computer science and security (IJCSS), vol. 4.

[33] J. Y. Kim, H. K. Choi, and S. Song, "A secure and lightweight approach for routing optimization in mobile IPv6," EURASIP Journal on Wireless Communications and Networking - Special issue on wireless network security, vol. 7, 2009.

[34] Supriya and M. Khari, "Mobile Ad Hoc Netwoks Security Attacks and Secured Routing Protocols: A Survey," Advances in Computer Science and Information Technology. Networks and Communications Lecture Notes of the Institute for Computer Sciences, Social Informatics and Telecommunications Engineering, vol. 84, pp. 119-124, 2012.

[35] J. Soryal and T. Saadawi, "IEEE 802.11 Denial of Service attack detection in MANET," Wireless Telecommunications Symposium (WTS), 2012.

[36] R. H. Jhaveri, S. J. Patel, and D. C. Jinwala, "DoS Attacks in Mobile Ad Hoc Networks: A Survey," presented at the Second International Conference on Advanced Computing \& Communication Technologies (ACCT), 2012 
[37] A. Michael and Nadeem, "Adaptive intrusion detection \& prevention of denial of service attacks in MANETs," presented at the IWCMC '09 Proceedings of the International Conference on Wireless Communications and Mobile Computing, Connecting the World Wirelessly, 2009.

[38] J. Su and H. Liu, "Protecting Flow Design for DoS Attack and Defense at the MAC Layer in Mobile Ad Hoc Network," Applied Informatics and Communication Communications in Computer and Information Science, vol. 224, pp. 233-240, 2011.

[39] A. Hamieh and J. Ben-othman, "Detection of Jamming Attacks in Wireless Ad Hoc Networks Using Error Distribution," presented at the International Conference on Communications, ICC '09. IEEE, 2009.

[40] J. Ben-othman and A. Hamieh, "Defending method against jamming attack in wireless ad hoc networks," presented at the 34th Conference on Local Computer Networks, LCN, IEEE, 2009.

[41] D. Glynos, P. Kotzanikolaou, and C. Douligeris, "Preventing impersonation attacks in MANET with multi-factor authentication," hird International Symposium on Modeling and Optimization in Mobile, Ad Hoc, and Wireless Networks, WIOPT, 2005.

[42] C. Douligeris, P. Kotzanikolaou, and D. Glynos, "Preventing Impersonation Attacks in MANET with Multi-Factor Authentication," WIOPT '05 Proceedings of the Third International Symposium on Modeling and Optimization in Mobile, Ad Hoc, and Wireless Networks, 2005.

[43] M. Barbeau, J. Hall, and E. Kranakis, "Detecting Impersonation Attacks in Future Wireless and Mobile Networks," Secure Mobile Ad-hoc Networks and Sensors Lecture Notes in Computer Science, vol. 4074, pp. 80-95, 2006.

[44] N. Dixit, S. Agrawal, and V. K. Singh, "A Proposed Solution for security Issues In MANETs," International Journal of Engineering Research \& Technology(IJERT), vol. 2, 2013.

[45] Vaithiyanathan, S. R. Gracelin, E. N. Edna, and S. Radha, "A Novel Method for Detection and Elimination of Modification Attack and TTL Attack in NTP Based Routing Algorithm," presented at the International Conference on Recent Trends in Information, Telecommunication and Computing (ITC), 2010

[46] P. Yi, X. Jiang, and Y. Wu, "Distributed intrusion detection for mobile ad hoc networks," Journal on Systems Engineering and Electronics, IEEE, vol. 19, 2008.

[47] S. R. Afzal, S. Biswas, J. B. Koh, T. Raza, and m. authors, "RSRP: A Robust Secure Routing Protocol for Mobile Ad Hoc Networks," presented at the Wireless Communications and Networking Conference,WCNC, IEEE, 2008.

[48] P. T. Tharani, K. Muthupriya, and C. Timotta, "Secured consistent network for coping up with gabrication attack in MANET," international journal of Emerging Technology and Advanced Engeineering, vol. 3, 2013.

[49] D. Sharma, P. G. Shah, and X. Huang, "Protecting from Attacking the Man-in-Middle in Wireless Sensor Networks with Elliptic Curve Cryptography Key Exchange," presented at the NSS '10 Proceedings of the Fourth International Conference on Network and System Security, 2010.

[50] K. Vishnu, "A new kind of transport layer attack in wireless Ad Hoc Networks," presented at the International Conference on Wireless Communications, Networking and Information Security (WCNIS), 2010

[51] X. Zou, A. Thukral, and B. Ramamurthy, "An Authenticated Key Agreement Protocol for Mobile Ad Hoc Networks," Mobile Ad-hoc and Sensor Networks Lecture Notes in Computer Science, vol. 4325, pp. 509-520, 2006.

[52] J. Liu, F. Fu, J. Xiao, and Y. Lu, "Secure Routing for Mobile Ad Hoc Networks," presented at the Eighth ACIS International Conference on Software Engineering, Artificial Intelligence, Networking, and Parallel/Distributed Computing, SNPD, 2007.

[53] J. Sen, B. Tata, M. Chandra, S. Harihara, and H. Reddy, "A mechanism for detection of gray hole attack in mobile Ad Hoc networks," presented at the 6th International Conference on Information, Communications \& Signal Processing, 2007

[54] G. Usha and S. Bose, "Impact of Gray hole attack on adhoc networks," presented at the International Conference on Information Communication and Embedded Systems (ICICES), 2013

[55] G. Xiaopeng and C. Wei, "A Novel Gray Hole Attack Detection Scheme for Mobile Ad-Hoc Networks," presented at the IFIP International Conference on Network and Parallel Computing Workshops, NPC Workshops, 2007.

[56] C. Gray, J. Byrnes, and S. Nelakuditi, "Pair-wise Resistence to traffic Analysis in MANETs," ACM SIGMOBILE Mobile Computing and Communications Review, 2008.

[57] E. A. Panaousis, T. A. Ramrekha, and C. Politis, "Secure routing for supporting ad-hoc extreme emergency infrastructures," Future Network and Mobile Summit, 2010. 
[58] M. Salmanian and M. Li, "Enabling secure and reliable policy-based routing in MANETs," presented at the military communications conference, MILCOM, 2012.

[59] F. R. Yu, H. Tang, S. Bu, and D. Zheng, "Security and quality of service (QoS) co-design in cooperative mobile ad hocnetworks," EURASIP Journal on Wireless Communications and Networking - Special issue on wireless network security, 2013.

[60] R. Gujral, A. Kapil, and ”", Volume , , pp "Secure QoS Enabled On-Demand Link-State Multipath Routing in MANETs," Information Processing and Management Communications in Computer and Information Science, vol. 70, pp. 250-257, 2010.

[61] A. El-Sayed, "Clustering Based Group Key Management for MANET," Advances in Security of Information and Communication, Networks Communications in Computer and Information Science, vol. 381, pp. 11-26, 2013.

[62] M. S. Zefreh, A. Fanian, S. M. Sajadieh, P.Khadivi, and M. Berenjkoub, "A Cluster-Based Key Establishment Protocol for Wireless Mobile Ad Hoc Networks," Advances in Computer Science and Engineering Communications in Computer and Information Science, vol. 6, pp. 585-592, 2009.

[63] L. Yingbin, H. V. Poor, and Y. Lei, "Secrecy Throughput of MANETs Under Passive and Active Attacks," Information Theory, IEEE Transactions on, vol. 57, pp. 6692-6702, 2011.

[64] W. El-Hajj, D. Kountanis, A. Al-Fuqaha, and M. Guizani, "A Fuzzy-Based Hierarchical Energy Efficient Routing Protocol for Large Scale Mobile Ad Hoc Networks (FEER)," in Communications, 2006. ICC '06. IEEE International Conference on, 2006, pp. 3585-3590.

[65] M. Achankunju, R. Pushpalakshmi, and A. A. Kumar, "Particle swarm optimization based secure QoS clustering for mobile ad hoc network," in Communications and Signal Processing (ICCSP), 2013 International Conference on, 2013, pp. 315-320.

\section{AUTHORS}

Ali Dorri received his B.S. degree in computer engineering from Bojnord University, Iran, in 2012, and now is student in M.S in software engineering in Mashhad branch, Islamic Azad University, Mashhad, Iran. His research interests cover Wireless Sensor Networks (WSN), Mobile Ad hoc Network (MANET) and specially Security challenges.

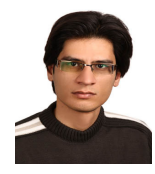

Dr. Seyed Reza Kamel Tabbakh is with the Department of Software Engineering, Faculty of Engineering, Islamic Azad University - Mashhad branch, Mashhad, Iran. He received his $\mathrm{PhD}$ in communication and network engineering from University Putra Malaysia (UPM) in 2011. He received his BSc and MSc in software engineering from Islamic Azad University, Mashhad branch and Islamic Azad University, South Tehran branch, Iran in 1999 and 2001 respectively. His research interests include IPv6 networks, routing and security. During his studies, he has published several papers in International journals and conferences.

Esmaeil Kheirkhah received his Bachelor and Master in Computer Science and Mathematics from Islamic Azad University, Mashhad, Iran in 1992 and 1996 respectively. $\mathrm{He}$ also received his $\mathrm{PhD}$ in Computer Science from National University of Malaysia (UKM) in 2010. He is currently an assistant professor at the Islamic Azad University of Mashhad. His research interests include the Software Engineering, Requirements Engineering, End-User Computing, and semantic-enabled software engineering.
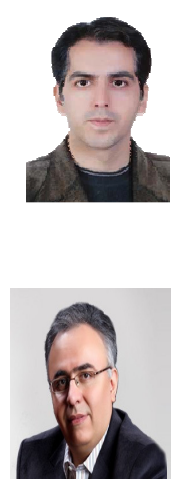